Estudios Románicos, Volumen 28, 2019, pp. 307-316

ISSN: 0210-491

eISSN: 1989-614X

DOI: https://doi.org/10.6018/ER/348571

\title{
ILUMINACIONES EN LA SOMBRA: EL DIARIO DE ALEJANDRO SAWA EN LA LITERATURA ESPAÑOLA*
}

(Iluminaciones en la sombra: the diary of Alejandro Sawa in Spanish literature)

\author{
Álvaro Luque Amo** \\ Universidad de Granada
}

\begin{abstract}
Iluminaciones en la sombra (1910) was published one year after the death of Alejandro Sawa (1862-1909). The present work proposes to analyze its character as a personal diary with a literary entity. This can affect the canonical consideration of this work. Iluminaciones en la sombra has been defined as a book of essays, a book of chronicles, a miscellaneous book or a novel; only some specialists have catalogued it as a personal diary. This article aims to clarify the literary and autobiographical nature of this work, with the objective of confirming its importance in the constitution of a new genre in the Spanish context.
\end{abstract}

Key Words: Sawa; Iluminaciones en la sombra; Personal Diary; Literary Diary; Theory of Autobiography; Spanish Literature.

Resumen: Iluminaciones en la sombra (1910) se publicó un año después de la muerte de Alejandro Sawa (1862-1909). El presente trabajo propone analizar su carácter de diario personal con entidad literaria, algo que no ha ocurrido con frecuencia y que puede ayudar a su asentamiento canónico. Se ha definido Iluminaciones en la sombra, así, como un libro de ensayos, un libro de crónicas, un libro misceláneo a secas e incluso como una novela, y solo algunos especialistas lo han catalogado como un diario. Un análisis que permita una definición más clara sobre su naturaleza literaria y autobiográfica puede dar cuenta de su importancia en la constitución de un nuevo género en el contexto español.

${ }^{*}$ Este trabajo se ha realizado bajo el patrocinio de una Ayuda FPU concedida por el Ministerio de Educación, Cultura y Deporte. Además, se enmarca dentro del proyecto de investigación I+D «PROCESOS DE SUBJETIVACION: BIOPOLITICA Y POLITICA DE LA LITERATURA. LA HERENCIA DEL ULTIMO M. FOUCAULT» (FFI2015-64217-P).

${ }^{* *}$ Dirección para correspondencia: Álvaro Luque Amo. Departamento de Lingüística General y Teoría de la Literatura. Facultad de Filosofía y Letras. Universidad de Granada. Campus Universitario de Cartuja. 18071 Granada (aluqueamo@ugr.es). 
Palabras clave: Sawa; Iluminaciones en la sombra; Diario personal; Diario literario; Teoría de la autobiografía; Literatura española.

Iluminaciones en la sombra (1910) se publicó un año después de la muerte de Alejandro Sawa (1862-1909). El presente trabajo propone analizar su carácter de diario personal con entidad literaria, algo que no ha ocurrido con frecuencia y que puede ayudar a su asentamiento canónico. Se ha definido Iluminaciones en la sombra, así, como un libro de ensayos, un libro de crónicas, un libro misceláneo a secas e incluso como una novela - González Martel, por poner un ejemplo reciente, habla de "memorias" para definir el libro en 2017 (González Martel, 2017: 117)—, y solo en escasas ocasiones algunos especialistas, como puede ser el caso de Iris M. Zavala (1977) o José Carlos Mainer (1986), lo han catalogado como un diario. Esto ha propiciado una confusión terminológica que, unida a la fragilidad de la figura de Sawa en el canon literario, ha sepultado esta obra' ${ }^{1}$. Además, y como ha sugerido ya un autor como Andrés Trapiello, es muy probable que se trate del "primer gran diario de intimidad literaria de la literatura moderna española" (Trapiello 2009: 22), lo que da cuenta de su importancia en la constitución de un nuevo género en el contexto español.

\section{Iluminaciones en la sombra (1910)}

\section{1. Su definición como diario personal}

Una de las definiciones más precisas de Iluminaciones en la sombra la ofrece ValleInclán, que lo cataloga como "un diario de esperanzas y tribulaciones" (Álvarez Hernández 1963: 71). Resultando evidente el tono sentimental y poético del texto, lo determinante es que Valle-Inclán no duda en calificarlo de diario. En esta misma línea, cuando Sawa publica algunos fragmentos de Iluminaciones en la revista Helios, lo hace bajo el título de Dietario de un alma (Zavala 1977: 57), lo que demuestra su propia intención. Es más, en la primera página del libro, Alejandro Sawa presenta su método:

Me he levantado temprano (...) y me he puesto a escribir estas hojas de mi dietario. Lo mismo me propongo hacer todos los días; luego repartiré mis jornadas en zonas de acción paralelas, aunque heterogéneas; y digo que paralelas, porque todas han de estar influidas por el mismo pensamiento que me llena por completo: la formación de mi personalidad (Sawa 2009: 37-38).

Ese pensamiento, la formación de su personalidad, es un objetivo recurrente en la práctica de llevar un diario personal. Sawa, además, explica que cada día va a escribir en

\footnotetext{
1 Una rápida mirada a los manuales de literatura española del siglo XIX y XX puede arrojar luz sobre este hecho. De los manuales consultados, varios de ellos no mencionan, siquiera, a Alejandro Sawa (Valbuena Prat 1983; Díez Borque 1980); otros hacen una sencilla mención a Sawa y a Iluminaciones, como es el caso de Mainer en los dos manuales en que colabora (1994; 2011) o Lina Rodríguez Cacho (2009). En una obra que está a medio camino entre el ensayo y el manual de historia de la literatura, Mainer (1986) sí desarrolla una gran entrada en el que supone el mayor acercamiento a Sawa desde el prólogo de Iris M. Zavala en la edición de 1977 y el de Andrés Trapiello en la de 2004 y 2009. Todo lo anterior demuestra que Iluminaciones en la sombra no ha sido contemplada por el canon literario español hasta fechas recientes y que durante el siglo XX ha sido generalmente ignorada.
} 
su cuaderno, con la rutina propia del diarista. En resumen, el modus operandi es el propio del diario, el tono es personal y cotidiano, el mismo Sawa reconoce escribir un dietario - término que, en este caso, no difiere del de diario -; si esto resulta tan claro, cabe preguntarse por qué durante tanto tiempo — con escasas excepciones como las citadas o la de críticos como José Esteban, que ya lo aventuró en $1978^{2}$ - ha habido críticos que no han sabido categorizar el libro, y la respuesta debe encontrarse en la escasez de estudios sobre escritura diarística en el contexto español. No se ha sabido con exactitud qué es un diario personal, y menos si este no ha tenido en el título el claro apelativo de diario.

Cuando el especialista se acerca con detenimiento a Iluminaciones en la sombra descubre, sin embargo, su idiosincrasia diarística. Si bien el libro es misceláneo en su naturaleza, con textos periodísticos y crítico-literarios, a lo largo de sus páginas predomina la entrada propia del diario personal y, lo que es más importante, el Yo de Sawa, que preside el desarrollo del libro. Se puede dividir Iluminaciones en la sombra en dos estilos de escritura diferenciados; en el primero, puramente diarístico, se recopilan pasajes autobiográficos y periodísticos; en el segundo, Sawa se inscribe en la tradición de las semblanzas a los autores admirados, al modo de un canon personal, tal y como es el caso de Darío y Los raros o Verlaine y su libro Los poetas malditos. Este último estilo de escritura se corresponde, de hecho, con los pasajes que el autor encuadró dentro de un ciclo de semblanzas emblemáticas denominado "De mi iconografía". Si bien en la edición original de 1910 (Sawa 1910) los pasajes diarísticos se entremezclan con las anteriores, en las dos últimas ediciones (Sawa 2004; 2009) el libro de Sawa ha sido dividido en dos partes: la primera, denominada como tal "Iluminaciones en la sombra", solo se ocupa del texto diarístico; la segunda, denominada "De mi iconografía", aglutina todos los retratos emblemáticos. Aunque es cierto que esta segunda parte no podría catalogarse de escritura diarística al uso, en este trabajo respetamos su inclusión en la obra, que contemplamos como un opus abarcador y total, porque en última instancia el diario no es sino una forma abierta.

Este último concepto de Beatrice Didier se basa en la tesis de que "dado que ese tipo de escritura no conoce reglas ni verdaderos límites, el diarista puede integrar en su texto las facturas de la lavandería, recortes de periódico (...) a fin de cuentas todo" (Didier 1996: 39). El diario se concibe así como un cuaderno total en el que, atendiendo a su origen sencillamente notarial, puede haber cabida para cualquier tipo de escritura. Esto explica la confusión creciente respecto de la obra de Sawa y la conjunción de estilos, géneros y escrituras en Iluminaciones. Esta forma abierta, sin embargo, tiene a su vez un contrapeso en la estructura diarística de la entrada, que Sawa respeta absolutamente. Todo el texto se ordena a partir de entradas diarias, e incluso una parte de ellas están fechadas, lo que deviene uno de los rasgos más característicos del diario, y en otros casos la marca temporal es reveladora: "Ayer una carta de Rubén Darío" (Sawa 2009: 49). En este sentido, resulta conveniente el concepto de Maurice Blanchot ${ }^{3}$, según el cual el diario está condicionado por una cláusula temporal definida por el calendario: el día. Así pues, lo escrito por Sawa se enmarca dentro de lo cotidiano, y la prueba fehaciente de ello es tiempo verbal empleado en sus páginas.

2 https://elpais.com/diario/1978/03/08/cultura/258159602 850215.html

3 Blanchot indica lo siguiente: "El diario íntimo, que parece tan desprendido de las formas (...) está sometido a una cláusula de apariencia liviana pero temible: debe respetar el calendario. Este es el pacto que sella. El calendario es su demonio, el inspirador, el compositor, el provocador y el guardia. Escribir su diario íntimo significa ponerse momentáneamente bajo el amparo de los días comunes" (Blanchot 1959: 207). 
Sawa habla siempre desde el presente, desde el que narra hechos recién ocurridos: "Recibo una invitación para asistir a una fiesta literaria" (Sawa 2009: 94) o "Acabo de conocer a un español bien educado" (46). Esto es lo que conduce a Genette a definir el diario, por la proximidad entre lo narrado y el momento de narración, como una narración intercalada ${ }^{4}$, algo que el texto de Sawa, como diario, comparte en gran parte de sus entradas.

$\mathrm{Si}$, en definitiva, desde un punto de vista formal es obvio que el texto de Sawa se rige por el formato del diario, el contenido, como se ha dejado entrever, reúne varios tipos de escritura. Sawa recopila así artículos periodísticos, reflexiones metaliterarias, frecuenta el aforismo o simplemente anota sus pensamientos íntimos. Todas estas escrituras forman parte del diario que se ha definido como forma abierta. Más importante que eso, en última instancia, es el desarrollo de un Yo diarístico. Este Yo, la construcción de la personalidad de Sawa, es la que define Iluminaciones en la sombra como un diario personal, lo que además ayuda a explicar su naturaleza literaria.

\subsection{Diario literario}

En el anterior punto se ha definido Iluminaciones en la sombra como un diario personal. De lo que se ha descrito, además, puede deducirse que el diario de Alejandro Sawa posee un tono privado que permite exponer la personalidad del Yo diarístico. Este tono se ha podido intuir en alguno de los extractos mencionados y en pasajes como este, en el que habla de la muerte de su padre:

Mi padre acaba de morir, hoy 16 de junio de 1905, a las once y diez minutos de la mañana: son las once y media. Mi mano está firme al escribir estas líneas y mis ojos secos. (...) Ahí está, en la alcoba de al lado, el cadáver de mi padre, y yo aquí, ante mi mesa, escribiendo estas líneas. Cuando se lo lleven para siempre, cuando lo pierda materialmente, entonces se asomará el dolor a mi boca y a mis labios. Ahora lo tengo aquí quieto en mi corazón, como una fiera amodorrada (Sawa 2009: 149).

Sawa, aunque desde los inicios muestre su intención de publicar el libro, y por tanto no escriba exclusivamente para sí mismo — si bien es algo que se prevé imposible —, respeta las convenciones del diario personal, e Iluminaciones en la sombra podría inscribirse sin lugar a dudas en la tradición del llamado diario íntimo.

Hasta el momento, en este trabajo se ha tratado al diario de diario personal, que es la denominación que nos interesa para lo que otros han preferido considerar como diario

4 Dice Genette que cabe entender el diario como una narración intercalada, en tanto que la proximidad entre historia y narración produce un efecto de roce entre "el desfase temporal del relato de acontecimiento («Hoy me ha ocurrido lo siguiente») y la simultaneidad absoluta en la exposición de los pensamientos y los sentimientos («Esta noche pienso lo siguiente»)"(Genette 1988: 275).

5 Trapiello señala: "En principio lo hacemos para nosotros mismos, pero nadie que lleve un diario ha renunciado a que pueda ser leído alguna vez por otro. A veces alguien concreto de quien se habla en sus páginas, a veces alguien abstracto, suma de todos esos lectores, o mejor, suma de todos esos seres a quien se ama de modo secreto mientras se escribe. Si toda la literatura es una declaración de amor, los diarios son una desesperada declaración de amor. Ni siquiera aquellos que han recurrido a sistemas complicados criptográficos como Samuel Pepys, o, entre nosotros, Cansinos Assens, autor de unos diarios inéditos de la guerra, ha renunciado a ello. Al contrario, se diría que tras de la criptografía lo que se busca es un lector mucho más agudo y comprometido, alguien en realidad dispuesto a compartir el secreto o si se prefiere alguien al que se pone a prueba" (Trapiello 1998: 28). 
intimo. Este último concepto, que surge tras la publicación del diario personal de Amiel por parte de E. Scherer, en 1883, ha tenido cierta relevancia en los estudios teóricos sobre la escritura diarística, si bien los diarios que se han publicado desde la segunda parte del siglo XX hasta ahora han dejado, por lo general, de titularse así, y desde nuestro punto de vista el término conduce a confusión; en este sentido, lo que muchos han definido como diario íntimo es lo que he denominado en otro lugar diario literario (Luque Amo, 2016): aquel texto diarístico que desarrolla un Yo capaz de ser leído como personaje y por tanto ser interpretado desde la literatura, respetando siempre lo que Lejeune bautizó como pacto autobiográfico (1994). Así, desde la aparición de los diarios de Samuel Pepys y Henri Frederic Amiel, y la consagración de la forma diarística en el siglo XIX, el diario ha reemplazado a su lector exclusivamente interesado en lo informativo por un lector que es capaz de leer el diario como una forma literaria, lo que ha propiciado su interpretación desde los estudios literarios y la búsqueda de estos por justificar el estatuto literario del diario, algo que sucede gracias al desarrollo de un Yo que puede ser leído como personaje.

Partiendo de esta base, es posible leer el diario de Alejandro Sawa como un texto literario. Iluminaciones en la sombra desarrolla así un Yo que es autobiográfico pero que al mismo tiempo puede leerse como personaje literario. El espacio privado mencionado al comienzo de este pasaje otorga profundidad a un Yo que va evolucionando desde su aparición en la primera frase del libro: "Quizá sea ya tarde para lo que me propongo: quiero dar batalla a la vida" (Sawa 2009: 37). Este Yo de Sawa se emparenta con los grandes personajes autobiográficos desde la aparición de Montaigne. En Iluminaciones en la sombra, además, aparece curiosamente un Sawa que nos resulta mucho más real que las descripciones del bohemio decadente que de él hacían Carrere, Valle-Inclán y otros autores de la época. Cuando Sawa describe episodios como el de su padre está mostrando un rostro que no recuerda el biógrafo, el panegirista, el que mitifica la figura del escritor después de muerto. En Iluminaciones en la sombra está el Yo privado de Sawa y también está el Yo público, y estas dos formas de una misma identidad conviven a lo largo del texto para formar el Yo diarístico que el lector interpreta como un personaje literario. De un modo similar lo explica Jordi Gracia cuando señala que "los diarios de algunos escritores se levantan como literatura gracias a esa materia particular que es el yo diluido" (Gracia 2004: 230). Iluminaciones en la sombra sobrevive al paso de las décadas no porque informe de la vida de alguien importante - ese Sawa ya no es frecuentador de la farándula literaria-, sino porque el autor es capaz de construir un Yo atractivo a ojos de un lector que se adentra en los recodos de su vida más de cien años después ${ }^{6}$. Un Yo que no es solo leído como autobiográfico, sino también como constructo literario; no solo como una individualidad, sino también como un tipo literario. En este sentido, la intimidad de la que se hablaba antes deviene en estas páginas intimidad literaria. Esta intimidad es, por tanto, un artefacto literario. Alguien como César Aira incide en esta línea de pensamiento: "Es bastante evidente que la creación de intimidad se parece mucho a la creación de literatura" (Aira 2008: 5). De esta manera, el diarista que describe los sucesos de su intimidad termina desarrollando un espacio textual que inevitablemente trasciende los moldes de lo real, para contener su propia idiosincrasia literaria.

6 Así lo entiende otra autora como Liliana Swiderski, quien publica uno de los escasos artículos dedicados íntegramente a Iluminaciones en la sombra: "Autorrepresentación y contexto cultural en Iluminaciones en la sombra" (2016). Allí estudia el carácter de autorrepresentación que tiene el texto de Sawa. 
En último lugar, cabe destacar el estilo literario del libro. Como los grandes poetas que publicaron poemarios bajo el título de diario —el caso paradigmático es el de Juan Ramón Jiménez y su Diario de un poeta recién casado - Sawa desarrolla este planteamiento a la inversa: crea un diario narrativo que sin embargo posee el tono y el estilo de un libro poético, lo que se puede comprobar en cualquiera de las entradas:

Hoy, 18 de junio, reanudo, mejor, reabro esta monótona exposición de horrores. Releyendo lo que antecede, me he creído en una trapería y no en un museo. Cuando las ilusiones se van, el cuerpo humano no es más que un almacén de podre. Niego y niego sistemáticamente, porque soy sincero. Mi vida no me da derecho a afirmar otra cosa sino el dolor. Mi perra prefiere sentarse sobre mi rodilla escuálida, a tomar el sol haciendo la rosca u ofreciendo sus ubres con voluptuosidad a las caricias del azul del cielo. Ella sabe lo que se hace. Yo tengo calor de soles en mi pecho para los que aman, y azul, mucho azul, con enormidades cerúleas, para los ingenuos que me ayudan en mi miseria y acomodan su vida a las mutaciones de mi alma (Sawa 1977: 111-112).

El estilo, cargado de figuras literarias como metáforas o símiles, arroja luz sobre la idiosincrasia del diario de Sawa, que se construye, como prosa lírica, mediante un tono similar al de la poesía en prosa y que es uno de los elementos que explican su naturaleza literaria.

\section{El diario personal en España: Sawa y el canon}

\subsection{Una panorámica}

Se ha sostenido tradicionalmente que la literatura autobiográfica no ha arraigado en territorio español como lo ha hecho en los países anglosajones o en Francia. Caballé señala:

En España, las escrituras autobiográficas nunca llegaron a generar una tradición, porque si por una parte la cultura hispánica fue de las primeras en volcarse con la autobiografía (el Diario de Ignacio de Loyola, el de Colón, las cuentas de conciencia o el Libro de la Vida de Teresa de Jesús, todos en el siglo XVI), esa inquietud renacentista por el individuo vino a coincidir con la Contrarreforma católica que fue una limitación brutal a la autoexpresión (Caballé 2015).

Esta tesis de Caballé puede explicar lo tardío de las primeras manifestaciones diarísticas en España, que es la forma que aquí se evalúa, pero más allá de la justificación interesa asumir desde un primer momento que el diario no se ha asentado en España hasta el siglo XX.

Los primeros diarios personales de los que se tiene constancia se mencionan en el extracto seleccionado: el diario de viaje de Colón, el diario espiritual de Ignacio de Loyola o los libros contables de los autores catalanes del siglo XVII. Estos diarios escapan, sin embargo, a la consideración de diario literario, en la medida en que están lejos de contener un Yo que se desarrolle en el texto diarístico. Con el paso de los años, a diferencia de lo que sucede en países como Francia, Inglaterra, Italia o Alemania, esta tendencia se 
mantiene, y los diarios más destacados del XVIII y el XIX no tienen la riqueza literaria de un Pepys o un Amiel. Sucede así en el caso de Fernández Moratín o en el de Jovellanos, que firman diarios en donde el tono es más propio de la crónica que del texto personal.

Pese a lo anterior, a mediados del XIX aparece en Inglaterra el Private Journal de José María Blanco White, uno de los personajes liberales de la España decimonónica. El diario de Blanco White presenta ya un espacio propio del diario moderno, si bien tanto el idioma del texto, escrito en inglés, como el hecho de que no se haya publicado hasta la fecha en España, propician la dificultad de considerar el diario desde la literatura española. A lo largo del XIX, se escriben otros diarios de cierto renombre como el de Miguel de Unamuno - aunque no se publica hasta 1970 - que sin embargo siguen sin ser textos susceptibles de ser leídos desde lo literario. De más importancia resulta la obra diarística de Azorín, que a finales del siglo XIX escribe una serie de libros protagonizados por una clara estructura diarística. Es el caso de Diario de un enfermo (1901) y Charivari, crítica discordante (1897). Esto provoca que, en términos de Trapiello (Trapiello 2009: 21), nos encontremos ante un autor que por fin comprende lo que es un diario al uso, si bien es cierto que estos textos carecen de un carácter autobiográfico, definiéndose más bien como novelas. Aunque algunos autores como el mismo Trapiello han querido ver en Azorín el primer diarista nacional, una especialista como Montserrat Escartín Gual señala lo siguiente:

Aunque no podemos afirmar que nuestro autor escribiera un diario personal, lo que está claro es su afición al género y a su estructura como vehículo literario desde sus primeros libros. Por ejemplo. Charivari, crítica discordante (1897) es un conjunto de artículos de crítica literaria que muestran, bajo la forma de un ficticio diario, las duras opiniones de José Martínez Ruiz sobre el mundo de las letras madrileño (Escartín 2003: 107).

Este uso de la escritura diarística va a ser una constante en Azorín, que partió de esta estructura para redactar su novela más famosa: La voluntad (1904). Sin embargo, estos textos están lejos de lo que en estas páginas se entiende por diario literario. Décadas después, superada la fecha de 1910 en la que Alejandro Sawa publica sus Iluminaciones, muchos escritores están escribiendo sus diarios, que publicarán a lo largo del siglo XX: es el caso de Rafael Cansinos Assens (1982), Josep Pla (1966), Emilio Prados (1966), Max Aub (1971), César González-Ruano (1970), Rosa Chacel (1982), Juan GilAlbert (1979) o Juan Larrea (1990) entre otros. Todos estos diarios, como se ve, son publicados en fechas muy tardías, de tal manera que no es posible ubicarlos, ni siquiera, en las coordenadas de Alejandro Sawa, que al escribir y publicar su diario se adelante varias décadas a esta época, como tratará de mostrarse a continuación.

\subsection{El primer diario literario en España: Iluminaciones y el canon literario}

Numerosos autores han incidido en esta idea: el diario personal es una forma fantasmal. Determinar su aparición, en la medida en que el diarista no escribía para ver publicada su obra y no existía un contexto - un mercado, un público - en el que esta se considerase publicable, resulta muy complicado, y además, lo que puede resultar aún más importante, plantea a los estudiosos del género la siguiente paradoja: si el diario perso- 
nal puede tardar años en publicarse, a veces siglos, se tienen que tener en cuenta para su estudio dos tipos de periodizaciones: la que valora el instante en que estos textos fueron escritos y la que, por otro lado, analiza la época de publicación de los mismos.

Hasta ahora, se ha seguido una cronología lineal basada en la creación del texto diarístico y solo se ha anotado las fechas de publicación de estos diarios como mera referencia. Si se tiene en cuenta, sin embargo, la publicación del diario de Leandro Fernández de Moratín (1867), los diarios ficcionales de Azorín (1897, 1901), el de Jovellanos (1915), el de Unamuno (1970), todos los mencionados en el anterior epígrafe (publicados de 1966 en adelante), se concluye, que el diario se asienta como forma en España en la segunda mitad del siglo XX. En 1963, Antonio Dorta y Manuel Granell publican la primera antología de diarios personales en español; de la década de 1970 en adelante empiezan a editarse con regularidad diaristas de todas las épocas; y a partir de la década de los ochenta (se publican grandes diarios como el de Gombrowicz) y noventa, surgen los grandes diaristas contemporáneos, caso de Trapiello, Sánchez-Ostiz o Iñaki Uriarte.

Puede deducirse, entonces, que la publicación del diario de Alejandro Sawa, en 1910, no es solo la publicación del primer diario literario, sino de uno de los primeros diarios personales en España. Esto le otorga importancia a la empresa llevada a cabo por Valle-Inclán y los más allegados de Sawa a principios de siglo. En este sentido, si se repasan todos los diarios mencionados hasta el momento, de los publicados o escritos antes de 1910 no hay ni uno solo que tenga la entidad literaria de Iluminaciones en la sombra, y que por tanto pueda inscribirse en la tradición de Samuel Pepys y de Amiel, que son los grandes precursores del diario como forma literaria. Alejandro Sawa es así un verdadero adelantado en la tradición hispánica, si bien este es un hecho más lógico de lo que cabría pensar en un primer momento, dada su afición por la literatura francesa e incluso su filiación a la tradición gala. De una literatura, la francesa, mucho más pródiga en escritura autobiográfica, Sawa heredó el gusto por la prosa diarística, y de ese gusto surgió la que él mismo catalogó como su obra fundamental.

En lo que concierne a la influencia posterior de Sawa, esta está limitada al reducido impacto de esa primera edición de 1910. Si bien la figura de Sawa está presente en el imaginario de todos los escritores noventayochistas e incluso de los autores cercanos a la Generación del 27, Iluminaciones en la sombra tiene un corto recorrido en el contexto literario de la época. Alguien como César González-Ruano, en una entrada de su diario, llega a decir lo siguiente:

Paso la tarde en casa releyendo Iluminaciones en la sombra, de Alejandro Sawa. Es un ejemplar de la primera edición que me ha regalado Hernán de Navascués. Lo ha comprado, por las buenas, en una librería de nuevo, y me dice que aún quedaban varios. La edición, a 3,50, es de Renacimiento y tiene como fecha 1910. Iluminaciones en la sombra, obra póstuma de Alejandro Sawa, es un gran libro. De él han hecho grandes ponderaciones los escritores más leídos y famosos del noventa y ocho. ¿Qué se pudo tirar entonces, en 1910, de este libro? ¿Mil ejemplares? Angustia pensar que en cuarenta y tres años no se han podido poner de acuerdo mil españoles para agotar esta edición (González-Ruano 1970: 408).

Aunque su biógrafa principal, Amelina Correa (2008), no especifica de cuánto fue esta tirada, Iris M. Zavala asegura que la intención inicial de Alejandro Sawa, que 
pensaba costear la edición d la obra en 1908, era publicar dos mil ejemplares, de tal manera que González-Ruano no anduvo muy desencaminado al escribir lo anterior. Si una edición de mil o dos mil ejemplares no se ha agotado a la altura de 1953, máxime cuando está prologado por alguien como Rubén Darío, puede aventurarse la recepción de un texto que rápidamente pasó a engrosar las filas de los marginados del canon.

De la afirmación de González-Ruano, no obstante, también puede colegirse el enorme respeto que sentían los escritores noventayochistas - encabezados por ValleInclán - por Sawa, y, lo que es más importante, su valoración de Iluminaciones en la sombra. Que alguien como González-Ruano, que publicó uno de los diarios más importantes del siglo XX español, defina la obra póstuma de Sawa como "un gran libro", puede arrojar luz sobre la huella que dejó el sevillano en los pocos escritores que se acercaron a la primera edición del libro.

En 1977, la editorial Alhambra, reedita Iluminaciones en la sombra con el mencionado prólogo de Iris M. Zavala, y la obra vuelve a cobrar vida, pero Sawa tampoco logra una resonancia que avale su escalada en el canon. Va a ser ya en 2004 y en 2009, al publicar Josef K. y Nórdica respectivamente las últimas ediciones que se conocen de Iluminaciones en la sombra, cuando se ratifique el ascenso de Alejandro Sawa y el espacio que empieza a ocupar en la literatura española. En el prólogo que comparten estas dos ediciones, Trapiello certifica el estatuto de Iluminaciones: "Raro libro este de la literatura española del novecientos, raro y valioso después de haber atravesado ya todos los purgatorios para convertirse en un clásico" (Trapiello 2009: 23).

\section{BIBLIOGRAFÍA}

AIRA, César (2008): "La intimidad". Boletín del Centro de Estudios de Teoría y Crítica Literaria. Vol. 13-14: 1-8.

ÁlVAREZ HERNÁNDEZ, Dictino (1963): Cartas de Rubén Dario. Madrid: Taurus. BLANCHOT, Maurice (1959): El libro que vendrá. Caracas: Monte Ávila.

CABALLÉ, Anna (2015): Entrevista con Eva Díaz Pérez: "El diario es un género que ha sido víctima de nuestra historia", Mercurio. Vol. 172: revistamercurio.es/secciones/entrevista/anna-caballe-el-diario-es-un-genero-que-ha-sido-victimade-nuestra-historia/ (29/11/2018).

CORREA RAMÓN, Amelina (2008): Alejandro Sawa: luces de bohemia. Marid: Fundación José Manuel Lara.

DIDIER, Beatrice (1996): "El diario ¿forma abierta?”. Revista de Occidente. Vol. 182183: 39-47.

DÍEZ BORQUE, José María (1980): Historia de la literatura española IV. Madrid: Taurus.

ESCARTÍN GUAL, Montserrat (2003): "El diario íntimo, Azorín y la nueva novela". Revista de Literatura. Vol. 127: 107-120.

GENETTE, Gerard (1988): Figuras III. Barcelona: Lumen.

GONZÁLEZ MARTEL, Juan Manuel (2017): "El ardiente epílogo de Iluminaciones en la sombra de Alejandro Sawa. Para la desconocida historia del original de una obra representativa de la bohemia literaria española". Revista de filología románica. Vol. 34.1: 117-134. 
GONZÁLEZ-RUANO, César (1970): Diario íntimo. Madrid: Taurus.

GRACIA, Jordi (2004): "La voz literaria y la materia del diarista", (Eds. María Ángeles Hermosilla Álvarez y Celia Fernández Prieto). Autobiografía en España, un balance. Madrid: Visor. 223-234.

LEJEUNE, Philippe (1994): El pacto autobiográfico. Madrid: Megazul.

LUQUE AMO, Álvaro (2016): "El diario personal en la literatura: teoría del diario literario". Castilla. Estudios de literatura. Vol. 7: 273-306.

MAINER, José-Carlos (1986): La edad de plata (1902-1939): ensayo de interpretación de un proceso cultural. Madrid: Cátedra.

(1994): Historia y crítica de la literatura española. Modernismo y 98. Barcelona: Crítica.

RODRÍGUEZ CACHO, Lina (2009): Manual de historia de la literatura española II. Madrid: Castalia.

SAWA, Alejandro (1910): Iluminaciones en la sombra. Madrid: Renacimiento.

(1977). Iluminaciones en la sombra. Madrid: Alhambra.

(2004). Iluminaciones en la sombra. Madrid: Josef K.

(2009): Iluminaciones en la sombra. Madrid: Nórdica.

SWIDERKSI, Liliana (2016): "Autorrepresentación y contexto cultural en Iluminaciones en la sombra". Dirāsāt Hispānicas: Revista Tunecina de Estudios Hispánicos. Vol, 3: 93-105.

TRAPIELLO, Andrés (1998): El escritor de diarios. Barcelona: Península. (2009) "Introducción", (Alejandro Sawa). Iluminaciones en la sombra. Madrid: Nórdica, 13-23.

VALBUENA PRAT, Ángel (1983): Del realismo al vanguardismo. Barcelona: Gustavo Gili.

ZAVALA, Iris María (1977): "Estudio preliminar", (Alejandro Sawa). Iluminaciones en la sombra. Madrid: Alhambra, 2-64.

\section{PERFIL ACADÉMICO Y PROFESIONAL}

Licenciado en Filología Hispánica por la Universidad de Córdoba (Premio Nacional de Fin de Carrera) y Máster en Estudios Literarios por la Universidad de Granada. Ha estado becado en el CCHS del CSIC y actualmente tiene un contrato FPU en el Departamento de Lingüística General y Teoría de la Literatura de la Universidad de Granada. Dentro de este proyecto, ha realizado estancias de investigación en Francia (Université de Pau) y Dinamarca (University of Copenhagen). Desde 2017 colabora además con el proyecto I+D "Procesos de subjetivación. Biopolítica y Política de la Literatura. El Último Foucault”. Sus líneas de investigación se centran en la teoría sobre escritura autobiográfica y se encuadran en el marco de la Teoría de la Literatura.

Fecha de recepción: 15/11/2018

Fecha de aceptación: 14/01/2019 